Vol. 48 (1993) [201-208]

\title{
STABILITY FOR SOME EQUATIONS OF MATHEMATICAL BIOLOGY AND MONOTONE FLOWS
}

\author{
IGOR V. FOMENKo
}

The theory of monotone flows and operators is applied to study stable equilibria of autonomous cooperative systems and stable periodic solutions of periodic perturbations of these systems. The connection between analyticity and the property of asymptotic stability is established.

The purpose of this paper is to apply the theory of monotone flows and operators recently developed in $[5,9,2]$ to some systems of mathematical biology. We shall study the existence of (asymptotically) stable equilibria for cooperative autonomous systems and the existence of (asymptotically) stable periodic solutions for their perturbations.

Before describing these systems we present one result for strongly monotone flows, which gives an answer to the following question.

On the one hand, it is well known $[9,2]$ that the result on the existence of a stable fixed point of a strongly monotone operator in an order interval may be extended to the existence of an asymptotically stable fixed point under the additional assumption of analyticity of this operator. On the other hand, the existence of stable equilibria in attractors of strongly monotone flows was obtained in [5]. What is the connection of existence of an asymptotically stable equilibrium and analyticity for strongly monotone flows?

\section{ASYMPTOTICALLY STABLE EQUILIBRIA IN STRONGLY MONOTONE FLOWS}

We use notations and definitions of [5]. Let $\phi$ be a strongly monotone flow in $\Omega \subset \mathbb{R}^{n}$. The set $\Omega$ is open and if $p_{1}, p_{2} \subset \Omega, p_{1} \ll p_{2}$ then

$$
\left[p_{1}, p_{2}\right]=\left\{z \in \mathbb{R}^{n} \mid p_{1} \leqslant z \leqslant p_{2}\right\} \subset \Omega .
$$

It follows from [5] that every attractor $\mathrm{K}$ for this flow contains a stable equilibrium.

TheOREM 1. Let the flow $\phi$ be analytic. If there exists an attractor $K$ then the flow $\phi$ has at least one asymptotically stable equilibrium.

Received 14th September, 1992

Research supported by an International Fellowship of the Natural Science and Engineering Research Council of Canada. The author wishes to thank L. Erbe, H.I. Freedman, J. Wu for their helpful suggestions.

Copyright Clearance Centre, Inc. Serial-fee code: 0004-9729/93 \$A2.00+0.00. 


\section{MODELS}

Many mathematical models in the biological sciences lead to the study of systems of differential equations

$$
\frac{d x_{i}}{d t}=x_{i} F_{i}(x),\left(x=\left(x_{1}, \ldots, x_{n}\right) \in \mathbb{R}_{+}^{n}, F_{i}(x) \in C_{1}\left(\mathbb{R}^{n}\right)(1 \leqslant i \leqslant n)\right)
$$

which have special properties.

We consider the following hypotheses.

H1. System (1) is dissipative (see [3]).

H2. System (1) is cooperative. This means

$$
\frac{\partial F_{i}}{\partial x_{j}} \geqslant 0 \quad(i \neq j ; i, j=1, \ldots, n)\left(x \in \text { int } \mathbb{R}_{+}^{n}\right) .
$$

We also require that the Jacobian matrix $\left[\left(\partial F_{i}\right) /\left(\partial x_{j}\right)\right]$ is irreducible for $x \in$ int $\mathbb{R}_{+}^{n}$ (see [5]).

H3. System (1) is a uniformly persistent system (see [1]).

H4. The functions $F_{i}$ are analytic in int $\mathbb{R}_{+}^{n}$.

TheOREM 2. Suppose (H1)-(H3) hold. Then system (1) has at least one stable equilibrium in int $\mathbb{R}_{+}^{n}$. If in addition the assumption $H 4$ holds then system (1) has at least one asymptotically stable equilibrium $x_{*} \in$ int $\mathbb{R}_{+}^{n}$.

Let

$$
\frac{d x_{i}}{d t}=x_{i}\left(F_{i}(x)+\varepsilon \varphi_{i}(t, x)\right), \quad\left(x \in \mathbb{R}_{+}^{n}\right)
$$

be the periodic perturbed system for the system (1).

H5. The vector-function $\varphi(t, x)=\left(\varphi_{1}(t, x), \varphi_{2}(t, x), \ldots, \varphi_{n}(t, x)\right)$ is smooth, periodic in $t$ of period $\omega$

$$
\varphi(t+\omega, x) \equiv \varphi(t, x) .
$$

Instead of hypothesis $\mathrm{H} 2$ we consider a stronger condition.

H6.

$$
\frac{\partial F_{i}}{\partial x_{j}}>0 \quad(i \neq j ; i, j=1, \ldots, n)\left(x \in \text { int } \mathbb{R}_{+}^{n}\right) .
$$

We also consider

H7. The vector-function $\varphi(t, x)$ is analytic.

TheOrem 3. Suppose H1, H3, H5, H6. Then there exists $\varepsilon_{0}>0$ such that for $\varepsilon \in\left(0, \varepsilon_{0}\right]$ system (2) has at least one stable w-periodic solution $x(t, \varepsilon) \in \operatorname{int} \mathbb{R}_{+}^{n}$.

Theorem 4. Suppose H1, (H3)-(H6) hold. Let $x_{*} \in$ int $\mathbb{R}_{+}^{n}$ be an asymptotically stable equilibrum of system (1), which exists by Theorem 1. Then for each $\delta>0$ 
there exists $\varepsilon(\delta)>0$ such that for each $\varepsilon \in(0, \varepsilon(\delta)]$ system (2) has a stable $\omega$-periodic solution $x(t, \varepsilon)$ and the inequality

$$
\left\|x(t, \varepsilon)-x_{*}\right\|<\delta
$$

holds.

ThEOREM 5. Let the conditions of Theorem 4 and $H 7$ hold. Then for each $\delta>0$ there exists $\varepsilon(\delta)>0$ such that for each $\varepsilon \in(0, \varepsilon(\delta)]$ system (2) has an asymptotically stable $\omega$-periodic solution $x(t, \varepsilon)$ and the inequality (3) holds.

\section{Proofs}

We consider $\mathbb{R}^{n}$ as the semiordered space with cone $\mathbb{R}_{+}^{n}$ (see [7]). We use the terminology of semiordered spaces

$$
x \geqslant y \Leftrightarrow x-y \in \mathbb{R}_{+}^{n}, x \gg y \Leftrightarrow x-y \in \operatorname{int} \mathbb{R}_{+}^{n} .
$$

For points $x_{-} \ll x_{+}$the set

$$
\left[x_{-}, x_{+}\right]=\left\{p \mid x_{-} \leqslant p \leqslant x_{+}\right\}
$$

is called the order interval.

The operator $A$ is a strongly monotone operator if for every two points $x_{1} \neq x_{2}$ belonging to its domain the inequality

$\begin{aligned} x_{1} & \geqslant x_{2} \\ A x_{1} & \gg A x_{2} .\end{aligned}$

Definition: Let $x_{*}$ be a fixed point of the operator $A$. Let the operator $A$ be defined in some neighbourhood of the point $x_{*}$. The point $x_{*}$ is called a stable fixed point of the operator $A$ if for each $\varepsilon>0$ there exists a $\delta>0$ such that the inequality $\left|x-x_{*}\right|<\delta$ implies the inequality $\left\|A^{n} x-x_{*}\right\|<\varepsilon(n=1,2, \ldots)$. If in addition

$$
\left\|A^{n} x-x_{*}\right\| \rightarrow 0 \quad(n \rightarrow+\infty)
$$

for points $x$ from some neighbourhood of the point $x_{*}$ then the point $x_{*}$ is called an asymptotically stable fixed point.

Proofs of Theorems 1-5 are based on the following statements, which are simple corollaries of results in $[\mathbf{9}, \mathbf{2}]$. 
THEOREM 6. Let the strongly monotone operator $A$ be defined in the order interval $\left[x_{-}, x_{+}\right]$. Let the inequalities

$$
A x_{-} \gg x_{-}, \quad A x_{+} \ll x_{+}
$$

hold. Then the operator $A$ has at least one stable fixed point $x_{*} \in\left[x_{-}, x_{+}\right]$.

THEOREM 7. Let the conditions of Theorem 6 hold. Let the operator $A$ be analytic in some neighbourhood of all its fixed points. Then there exists at least one asymptotically stable fixed point $x_{*} \in\left[x_{-}, x_{+}\right]$.

Proof of Theorem 1: Let the conditions of Theorem 1 hold. Let $K_{0}$ be the set of non-wandering points which belong to $K$. The set $K_{0}$ is nonempty. Fix $z \in K_{0}$. There exists a maximal element $p_{1} \in K_{0}$, a minimal element $p_{2} \in K_{0}, p_{1} \geqslant p_{2}$. It follows from [5] that the point $p_{1}$ is an asymptotically upper stable equilibrium and the point $p_{2}$ is an asymptotically lower stable equilibrium. If $p_{1}=p_{2}$ then the point $p_{1}$ is an asymptotically stable equilibrium. Suppose $p_{1} \neq p_{2}$. Then $p_{1} \ll p_{2}$ because the flow $\phi$ is strongly monotone. By [5], there exist $y_{2} \ll p_{2}, p_{1} \ll y_{1}\left(y_{1}, y_{2} \in \Omega\right)$ such that $y_{1}(t) \rightarrow p_{1}, y_{2}(t) \rightarrow p_{2}$. So there exists $T>0$ such that the following inequalities hold

$$
y_{1} \ll \phi(T) y_{1} \ll p_{1} \ll p_{2} \ll \phi(T) y_{2} \ll y_{2} .
$$

Since the operator $A=\phi(T)$ is analytic [8], by Theorem 7, it has an asymptotically stable fixed point $x_{*} \in\left[y_{1}, y_{2}\right]$. Hence the point $x_{*}$ belongs to the asymptotically stable periodic solution of system (1). But it is well known [4] that this solution has to be an equilibrium. Theorem 1 has been proved.

Proof of Theorem 2: Theorem 2 follows from [5, Theorem 4.1] and Theorem 1 for the attractor

$$
K=\coprod_{x \in \text { int }} \Lambda_{+}^{+} \Lambda^{+}(x)
$$

where $\Lambda^{+}(x)$ is the omega limit set of the orbit through $x$.

Proof of Theorem 3: Let the maximal element $p_{1} \in K_{0}$ and the minimal element $p_{2} \in K_{0}, p_{2} \leqslant p_{1}$ be as in the proof of the Theorem 3. The points $p_{1}$ and $p_{2}$ are asymptotically upper and lower stable equilibria, respectively.

Lemma 1. There exist two points $x_{-}, x_{+} \in$ int $\mathbb{R}_{+}^{n}$ such that the inequality

$$
x_{-} \ll \phi(\omega) x_{-} \ll p_{2}<p_{1} \ll \phi(\omega) x_{+} \ll x_{+}
$$

holds. 
Proof of Lemma 1: We prove the existence of an element $x_{+}$such that the inequality

$$
p_{1} \ll \phi(\omega) x_{+} \ll x_{+}
$$

holds.

Let $O\left(\delta_{0}, p_{1}\right)=\left\{p \mid\left\|p-p_{1}\right\|<\delta_{0}\right\}$ for some $\delta_{0}>0$ be a neighbourhood of the point $p_{1}$ such that for each $x \in O\left(\delta_{0}, p_{1}\right)$ the condition

$$
\phi(t) x \rightarrow p_{1}
$$

holds. Krasnoselskii's Theorem about eigenvectors [7] implies the existence of the point $x_{+} \in O\left(\delta_{0}, p_{1}\right) \cap\left(p_{1}+\right.$ int $\left.\mathbb{R}_{+}^{n}\right)$ such that

$$
\phi(\omega) x_{+}=\lambda\left(x_{+}-p_{1}\right)+p_{1},
$$

where $\lambda>0$.

Suppose $\lambda \geqslant 1$. Then $\phi(\omega) x_{+} \geqslant x_{+}$. Since $\phi(\omega)$ is a strongly monotone operator we have $\phi(n \omega) x_{+} \geqslant x_{+} \quad(n=1,2, \ldots)$. This inequality contradicts the condition (7). Hence the inequality (6) holds.

By the same reasoning the existence of the element $x_{-}$may be obtained.

Let

$$
G=\left\{\phi(t) x \mid x \in\left[x_{-}, x_{+}\right], t \in[0, \omega]\right\} .
$$

The set $G$ is compact and $G \subset \operatorname{int} \mathbb{R}_{+}^{n}$. Choose a compact set $G_{0} \subset \operatorname{int} \mathbb{R}_{+}^{n}$ such that $G \subset G_{0}$. Choose $\varepsilon_{1}>0$ such that inequalities

$$
x_{i}\left(\frac{\partial F_{i}}{\partial x_{j}}+\varepsilon \frac{\partial \varphi_{i}(t, x)}{\partial x_{j}}\right)>0 \quad\left(0<\varepsilon \leqslant \varepsilon_{1}, x \in G, t \in[0, \omega], i=1, \ldots, n\right)
$$

hold. Such an $\varepsilon_{1}>0$ exists due to H6.

By the theorem of continuation of solutions [4], there exists $\varepsilon_{2} \in\left(0, \varepsilon_{1}\right]$ such that

$$
p(t, x, \varepsilon) \in G_{0}\left(x \in\left[x_{-}, x_{+}\right], \varepsilon \in\left(0, \varepsilon_{2}\right], t \in[0, \omega]\right),
$$

where $p(t, x, \varepsilon)$ is the solution of system $(2), p(0, x, \varepsilon)=x$. Let $U(\omega, \varepsilon)$ be the operator of translation along trajectories of system (2)

$$
U(\omega, \varepsilon) x=p(\omega, x, \varepsilon) \quad\left(x \in\left[x_{-}, x_{+}\right], \varepsilon \in\left(0, \varepsilon_{2}\right], t \in[0, \omega]\right) .
$$

The operator $U(\omega, \varepsilon)$ is a strongly monotone operator in $\left[\boldsymbol{x}_{-}, \boldsymbol{x}_{+}\right]$(see [6]). It follows from (5) and the theorem of continuation of solutions [4] that there exists an $\varepsilon_{0} \in\left(0, \varepsilon_{2}\right]$ for which the inequality

$$
x_{-} \ll U(\omega, \varepsilon) x_{-} \ll p_{2} \ll U(\omega, \varepsilon) x_{+} \ll x_{+} \quad\left(\varepsilon \in\left(0, \varepsilon_{0}\right]\right)
$$


holds. Applying Theorem 6 to $\left[x_{-}, x_{+}\right]$and $A=U(\omega, \varepsilon)\left(\varepsilon \in\left(0, \varepsilon_{0}\right]\right)$ we obtain the existence of a stable fixed point for the operator $U(\omega, \varepsilon)\left(\varepsilon \in\left(0, \varepsilon_{0}\right]\right)$ in $\left[x_{-}, x_{+}\right]$. This proves Theorem 3.

Proof of Theorems 4, 5: Let the conditions of Theorem 4 hold. Let $x_{*}$ be an asymptotically stable equilibrium of system (1), which exists by Theorem 2 .

LEMma 2. For each $\delta$-neighbourhood $O\left(\delta, x_{*}\right)=\left\{p \mid\left\|p-x_{*}\right\|<\delta\right\}(\delta>0)$ there exist points $p_{-}, p_{+} \in O\left(\delta, x_{*}\right)$ such that the inequality

$$
p_{-} \ll \phi(\omega) p_{-} \ll x_{*} \ll \phi(\omega) p_{+} \ll p_{+}
$$

holds.

Proof of Lemma 2: The reasoning is the same as in the proof of Lemma 1.

Due to $\mathrm{H} 6$ we may choose $\varepsilon_{1}>0, \delta>0$ such that for $\varepsilon \in\left(0, \varepsilon_{1}\right]$ and for the functions

$$
\Phi_{i}(x, t)=x_{i}\left(F_{i}\left(x_{1}, \ldots, x_{n}\right)+\varepsilon \varphi_{i}(t, x)\right), \quad(i=1, \ldots, n)
$$

the inequalities

$$
\frac{\partial \Phi_{i}}{\partial x_{j}}>0 \quad\left(x \in O\left(\delta_{1}, x_{*}\right), i \neq j, i, j=1, \ldots, n\right)
$$

hold.

Lemma 3. There exist $\varepsilon_{2} \in\left(0, \varepsilon_{1}\right], \delta_{2} \in\left(0, \delta_{1}\right]$ such that the solution $p(t, x, \varepsilon)$ of system (2) $\left(p(0, x, \varepsilon)=x, \varepsilon \in\left(0, \varepsilon_{2}\right], x \in O\left(\delta_{2}, x_{*}\right)\right)$ is defined for all $t \in[0, \omega]$ and

$$
p(t, x, \varepsilon) \in O\left(\delta_{1}, x_{*}\right) \quad\left(x \in O\left(\delta_{2}, x_{*}\right)\right) .
$$

Proof of Lemma 3: Since the point $x_{*}$ is an equilibrium of system (1), this statement follows from the Theorem of continuation of solutions [4].

Now consider the operator $U(\omega, \varepsilon)$, which is defined by the formula

$$
U(\omega, \varepsilon) x=p(\omega, x, \varepsilon) \quad\left(x \in O\left(\delta_{2}, x_{*}\right)\right) .
$$

It follows from [6] that this operator is a strongly monotone operator in $O\left(\delta_{2}, x_{*}\right)$.

Lemma 4. Let the numbers $\varepsilon_{2}>0, \delta_{2}>0$ be choosen according to Lemma 3. For each $\delta \in\left(0, \delta_{2}\right)$ we may choose $\varepsilon(\delta) \in\left(0, \varepsilon_{2}\right]$ such that in the neighbourhood $O\left(\delta, x_{*}\right)$ there exist two points $x_{-}, x_{+}$for which the following condition

$$
x_{-} \ll U(\omega, \varepsilon) x_{-} \ll x_{+} \ll U(\omega, \varepsilon) x_{+} \ll x_{+}
$$


holds.

Proof of Lemma 4: For each $\delta \in\left(0, \delta_{2}\right)$ we choose points $p_{-}, p_{+}$such that (8) holds. Let $x_{-}=p_{-}, x_{+}=p_{+}$. Lemma 4 follows from the inequality (8) and the theorem of continuation of solutions [4].

Theorem 4 follows from Lemma 4 and Theorem 6. If $\mathrm{H} 7$ holds then the operator $U(\omega, \varepsilon)$ is analytic [8]. So Theorem 5 follows from Lemma 4 and Theorem 7.

\section{REFERENCES}

[1] G. Butler, H. Freedman and P. Waltman, 'Uniformly persistent systems', Proc. Amer. Math. Soc. 86 (1986), 425-430.

[2] E.N. Dancer and P. Hess, 'Stability of fixed points for order-preserving discrete-time dynamical system', J. Reine Agnew. Math. 419 (1991), 125-139.

[3] J.K. Hale, Asymptotic behavior of dissipative systems (American Mathematical Society, Providence, Rhode Island, 1988).

[4] J.K. Hale, Ordinary differential equations (Wiley-Interscience, New York, 1962).

[5] M.W. Hirsch, 'The dynamical system approach to differential equations', Bull. Amer. Math. Soc. 11 (1984), 1-64.

[6] M.W. Hirsh, 'Systems of differential equations which are competitive or cooperative II. Convergence almost everywhere', SIAM J. Math. Anal. 196 (1985), 423-439.

[7] M.A. Krasnoselskii, Positive solutions of operator equations (Noordhoff, Groningen, 1964).

[8] S. Lefschetz, Differential equations: geometrical theory (Wiley-Interscience, New York, 1957).

[9] A.V. Pokrovskii, 'Existence and calculation stable regimes in nonlinear systems', $A$ vtomat. Telemekh 4 (1986), 16-24.

Department of Mathematics

University of Alberta

Edmonton

Canada T6G 2G1 